\title{
A cross sectional clinicomycological study of dermatophytosis in a tertiary care hospital, North Karnataka, India
}

\author{
Sumathi. S ${ }^{1}$, Vishalakshi S Pandit ${ }^{2, *}$, Sangeetha Patil ${ }^{3}$, Vijayakumara Adavi ${ }^{4}$ \\ ${ }^{1}$ Associate Professor, ${ }^{2,3}$ Assistant Professor, ${ }^{4}$ Senior Resident, ${ }^{1,3}$ Dept. of Microbiology, ${ }^{2,4}$ Dept. of Dermatology, Koppal Institute
} of Medical Sciences, Karnataka, India

*Corresponding Author:

Email: vishalaxisp@yahoo.in

\begin{abstract}
Introduction: Dermatophytosis is superficial fungal infection caused by a group of keratinophilic fungi called dermatophytes capable of invading keratinized tissue of skin, hair and nail. The dermatophytes which cause human disease are divided into three main genera based on their morphological characteristics as Trichophyton, Microsporum and Epidermophyton. Hence, to determine the prevalence of dermatophytosis and to isolate different dermatophyte species.

Material and Methods: The present study was hospital based, cross-sectional study which was done in a tertiary care hospital. A total of 150 samples were collected from clinically suspected cases of dermatophytosis. Samples were subjected to mycological studies like KOH examination and fungal culture using Sabouraud Dextrose Agar (SDA) media with cycloheximide and chloramphenicol. Species were identified based on colony morphology, microscopic features using lactophenol cotton blue mount and confirmation by using slide culture. Whenever necessary, urease test was also done for species identification.

Results: In present study, out of 150 samples, majority of the patients were in1-10 yrs followed by 21-30 and 31-40 years age group and most of them were males. Among 150 samples collected, 96 (64\%) cases were KOH positive and 49 (33\%) were culture positive. Tinea corporis was the most common clinical type seen followed by tinea capitis, tinea cruris, and tinea faciei. Dermatophytes isolated which include Trichopyton mentagrophytes, T. rubrum, T. verrucosum, T. Schoenleinii, T. tonsurans, Microsporum gypseum, and Epidermophyton floccosum.

Conclusion: Tineacorporis was the commonest clinical type of dermatophytosis and $\mathrm{T}$. mentagrophytes was the most common species isolated in this study.
\end{abstract}

Keywords: Dermatophytosis, Trichophyton mentagrophytes, Trichophyton rubrum, Microsporum gypseum Slide culture.

\section{Introduction}

Cutaneous dermatophyte infections are common in the general population; upto $20 \%$ of people are infected at any time. Dermatophytes are closely related keratinophilic fungi with ability to degrade keratin and invade the skin, hair and nails, thus causing dermatophytosis (ring worm or tinea). ${ }^{1}$ Infection is generally cutaneous and restricted to the nonliving cornified layers because the fungi is not able to penetrate the deeper tissue or organ of healthy immunocompetent host. The infection is commonly designated as ring worm or "tinea". Tinea literally refers to insect larva (cloth moth) that was felt by Romans to be the cause of infection. Dermatophytes are moulds belonging to the three genera of fungi imperfecti (1) Microsporum, (2) Trichophyton and (3) Epidermophyton. ${ }^{2}$

Dermatophytes can infect humans when exposed to a contagious source. Risk factors include genetic predisposition to infection, humidity, heat, diabetes, prolonged use of glucocorticoids, unventilated shoes, poor hygiene or wet feet. Dermatophytes in the hair can be transmitted from contaminated combs, sprays or oils. ${ }^{3}$ The classical presentation of tinea infection is an annular lesion, sharply marginated with central clearing and surrounded by an advancing, inflamed, raised border. However, there is wide variation in clinical presentation depending upon the infecting species, size of inoculum, site of infection and immune status of the host. ${ }^{1}$

Though dermatophytes generally do not cause serious and life threatening infections, they cause temporary cosmetic disfigurement and associated itching. Distribution of dermatophytes varies with geographical regions and with time in the same geographical region. ${ }^{4}$ The clinical presentation is very often confused with other skin disorders due to application of steroids, making laboratory diagnosis and confirmation necessary. ${ }^{5}$

Knowing the prevalence and identifying the etiological agents of dermatophytosis is essential for providing the control measures. So, this study was conducted to know the prevalence and clinical pattern of dermatophyte infections in patients attending Dermatology outpatient department in a tertiary care centre.

\section{Material and Methods}

The study population included 150 patients, clinically suspected as having dermatophytosis randomly selected from the dermatology outpatient department of Koppal Medical college hospital Koppal from August 2015 to July 2016.

The samples like skin scrapings, hair and nails were collected in the department of microbiology from clinically suspected cases of dermatophytoses and 
subjected for direct microscopy in $\mathrm{KOH}$ and culture. The skin scrapings were collected from the active growing edges of the lesions with a blunt sterile scalpel, after cleaning the site with $70 \%$ alcohol and allowing it to dry. The infected nails were clipped and hair epilated after cleaning the infected site with $70 \%$ alcohol. Samples were processed as per standard mycological techniques.150 samples were subjected to direct microscopy in $10 \% \mathrm{KOH}$ for skin samples and $20 \%$ to $40 \% \mathrm{KOH}$ for hair and nail samples were inoculated into, two antibiotic incorporated SDA tubes with and without cycloheximide500 $\mathrm{mg} / \mathrm{l}$. SDA tube with cycloheximide is used to prevent the growth of saprophytic fungi. SDA tubes were incubated at room temperature $24^{\circ} \mathrm{c}$ to $30^{\circ} \mathrm{c}$ for upto four weeks. SDA tubes were observed for appearance of growth. As growth appears culture tubes were examined for colony morphology and pigment production. Dermatophyte species were identified based on colony morphology, pigment production and microscopic appearance of the hyphae and the appearance of macroconidia and microconidia and special structures like spiral hyphae, antler hyphae, pectinate hyphae, nodal or racquet hyphae. Slide cultures were prepared for identification of specific fungal species.

The microscopic examination of fungal growth was observed with Lacto Phenol Cotton Blue mount. Nature of mycelium, presence or absence of microconidia ,shape of macroconidia, pencil shaped, cigar like, balloon like and nature of their cell walls echinulated or smooth, number of septa or absence of conidia(micro or macroconidia) with presence of antler like hyphae, chlamydoconidia in chains were observed in the identification of various species of dermatophytes. Urease test was also performed to differentiate Trichophyton species.

\section{Statistical analysis}

Descriptive statistics such as mean, SD and percentage was used to present the data. Comparison between $\mathrm{KOH}$ mounts and culture was done by using chi-square test. Further, sensitivity, specificity, positive predictive value and negative predictive value were also calculated. A p-value less than 0.05 were considered as significant.

\section{Results}

A total of $150 \mathrm{pts}$ were enrolled in the study comprising $110(73 \%)$ males and $40(27 \%)$ females. Majority of cases were seen in the age group of 1- 10 years, followed by $21-30$ and 31 to 40 yrs. Tinea corporis was the most common clinical type followed by T. capitis. Overall positivity by culture was 49 (33\%) and by direct microscopy (KOH mount) 96 (64\%). In 46 (31\%) cases both $\mathrm{KOH}$ and culture were found to be positive.

In the present study, all the three genera of dermatophytes that is, Trichophyton, Epidermophyton, Microsporum were isolated as causative agents of infection .Of the total types of 7 different dermatophyte species isolated T.mentagrophytes was isolated in highest number of cases followed by T.rubrum, T.verrucosum, T.tonsurans, T.schoenleinii, E.floccosum, M.gypseum.

Out of 150 clinically suspected cases of dermatophytoses, fungi were demonstrated in 96 cases by direct microscopy and by culture in 49 cases. 46 cases were positive by both microscopy and culture. 50 cases $(33 \%)$ were positive by microscopy and negative by culture. $3(2 \%)$ cases were negative by microscopy but culture positive. 51 cases $(34 \%)$ were negative both by microscopy and culture.

Table 1: Age and sex distribution of the dermatophytosis cases

\begin{tabular}{|c|c|c|c|}
\hline Age group (yrs) & Male (\%) & Female (\%) & Total (\%) \\
\hline $1-10$ & $35(81.4)$ & $8(18.6)$ & $43(28.7)$ \\
\hline $11-20$ & $14(63.6)$ & $8(36.4)$ & $22(14.7)$ \\
\hline $21-30$ & $22(64.7)$ & $12(35.3)$ & $34(22.7)$ \\
\hline $31-40$ & $22(75.9)$ & $7(24.1)$ & $29(19.3)$ \\
\hline $41-50$ & $5(62.5)$ & $3(37.5)$ & $8(5.3)$ \\
\hline $51-60$ & $9(81.8)$ & $2(18.2)$ & $11(7.3)$ \\
\hline Above 60 & $3(100)$ & -- & $3(2.0)$ \\
\hline Total & $110(73.3)$ & $40(26.7)$ & 150 \\
\hline
\end{tabular}

Out of the 150 cases, majority of patients (28.7\%) were in the age group of 1-10 years followed by 21-30yrs (22.7\%) and 31- 40 yrs (19.3\%) and males(73.3\%) were more than females(26.7\%). 
Table 2: Clinical presentation of dermatophytosis

\begin{tabular}{|c|c|c|c|c|c|c|c|c|}
\hline $\begin{array}{l}\text { Clinical type of } \\
\text { dermatophyte } \\
\text { infection }\end{array}$ & 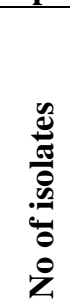 & 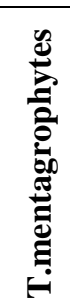 & مُ & 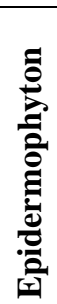 & 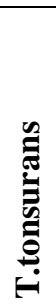 & 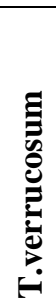 & 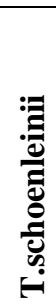 & \\
\hline T.corporis & 18 & 10 & 7 & - & - & - & - & 1 \\
\hline T.cruris & 8 & 4 & 4 & - & - & - & - & - \\
\hline T.unguium & 3 & 1 & 2 & - & - & - & - & - \\
\hline T.capitis & 13 & 5 & 1 & 1 & 2 & 3 & 1 & - \\
\hline T.pedis & 3 & 1 & 2 & - & - & - & - & - \\
\hline T.faciei & 2 & 2 & 0 & - & - & - & - & - \\
\hline T.manuum & 2 & 0 & 2 & - & - & - & - & - \\
\hline Total culture positive & 49 & 23 & 18 & 1 & 2 & 3 & 1 & 1 \\
\hline
\end{tabular}

In our study, of the different clinical types of dermatophytosis, following species of dermatophytes were isolated from different sites as shown in Table No. 2. Among these, common clinical type was $\mathrm{T}$ corporis (18) followed $\mathrm{T}$ capitis (13), $\mathrm{T}$ cruris (8) and so on. Cultures isolated from $\mathrm{T}$ corporis consists of T.mentagrophytes from 10 cases, T.rubrum from 7 cases and M.gypseum from 1 case. From 8 T.cruris cases T.mentagrophytes was isolated from 4 cases and T.rubrum from 4 cases. Cultures isolated from 13 cases of T.capitis include, T.mentagrophytes from 5 cases, T.verrucosum from 3 cases, T.tonsurans from 2 cases, T.rubrum from 1case, Epidermophyton from 1 case and T.schoenleinii from 1case.

Table 3: Comparison of $\mathrm{KOH}$ mounts and culture

\begin{tabular}{|l|c|c|c|c|c|}
\hline & KOH positive & KOH negative & Total & \multirow{2}{*}{$\boldsymbol{\chi}^{2}$ value } & p-value \\
\hline Culture positive & $46(31 \%)$ & $3(2 \%)$ & 49 & & \multirow{2}{*}{26.3} \\
\cline { 1 - 4 } Culture negative & $50(33 \%)$ & $51(34 \%)$ & 101 & \multirow{2}{*}{20001} \\
\hline Total & $96(64 \%)$ & $54(36 \%)$ & 150 & & \\
\hline
\end{tabular}

Sensitivity $=47.9 \%$, Specificity $=94.4 \%, \mathrm{PPV}=93.9 \%, \mathrm{NPV}=50.5 \%$

Table 4: Dermatophyte species isolated

\begin{tabular}{|l|c|c|}
\hline $\begin{array}{c}\text { Dermatophyte species } \\
\text { isolated }\end{array}$ & NNO & Percentage \\
\hline T.mentagrophytes & 23 & $46.9 \%$ \\
\hline T.rubrum & 18 & $36.7 \%$ \\
\hline T.verrucosum & 03 & $6 \%$ \\
\hline T.tonsurans & 02 & $4 \%$ \\
\hline T.schoenleinii & 01 & $2 \%$ \\
\hline E.floccosum & 01 & $2 \%$ \\
\hline M.gypseum & 01 & $2 \%$ \\
\hline Total culture positive & 49 & ---- \\
\hline
\end{tabular}

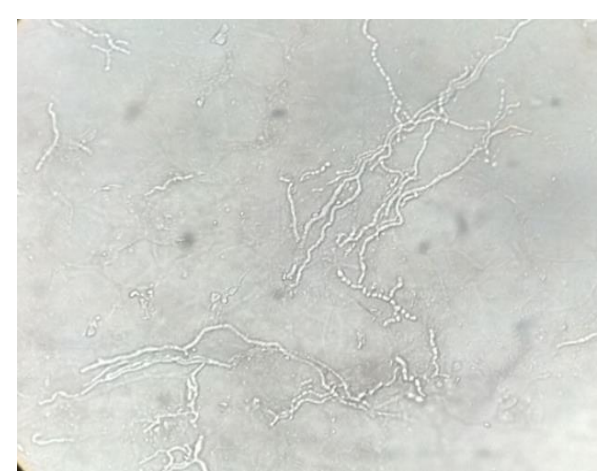

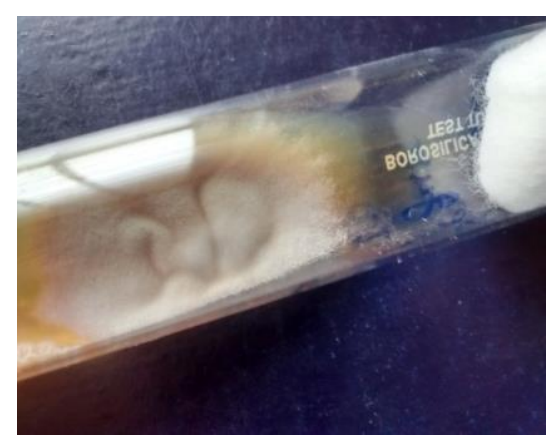

Fig. 2: Growth of Trichophyton. Spp on SDA 


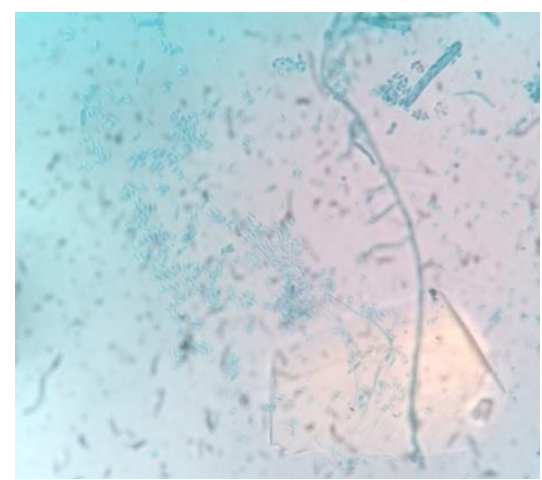

Fig. 3: Tricophyton rubrum LPCB mount

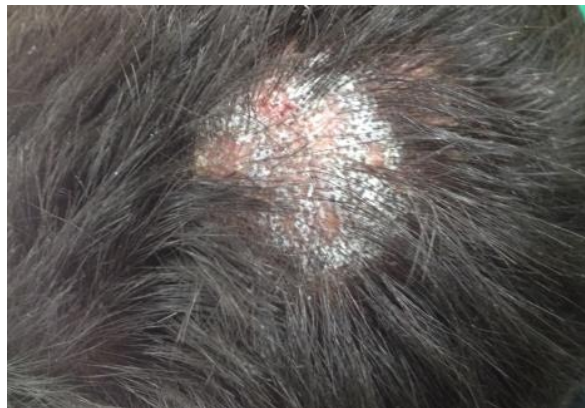

Fig. 4: Tinea capitis

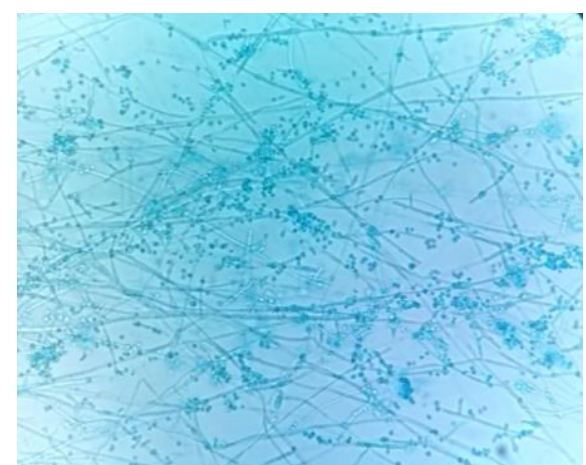

Fig. 5: Trichophyton mentagrophytes

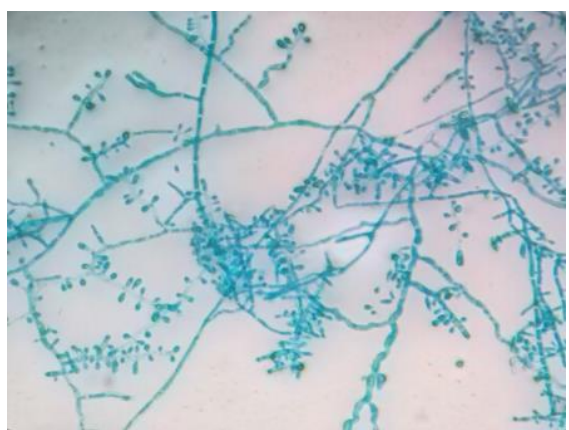

Fig. 6: Trichophyton tonsurans. LPCB mount from slide culture

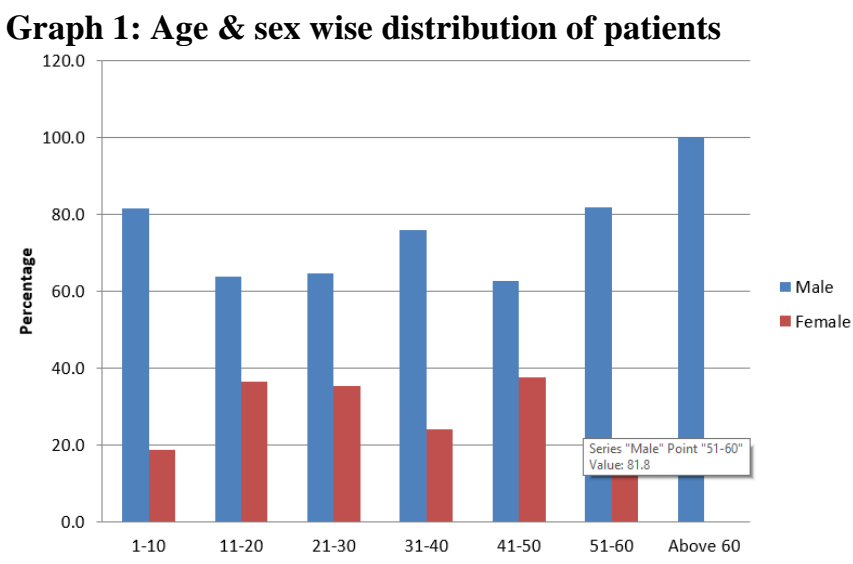

\section{Graph 2: Percentage of species isolated}

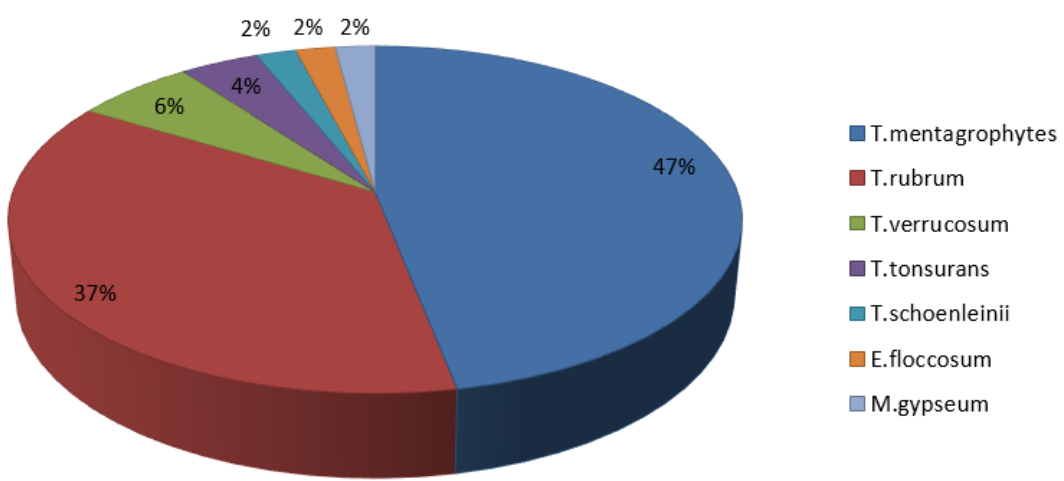

In this study T.mentagrophytes is the commonest isolate and of the 23 isolates of T. mentagrophytes, 10 were isolated from T.corporis, 5 from T.capitis, 4 from T.cruris, 2 from T.faciei, 1 each from T. unguium and
T.pedis. T.rubrum is the next common isolate and of the 18 isolates of T.rubrum, 7 were isolated from T.corporis, 4 from T.cruris, 2 from T.unguinum, 2 from T.pedis, 2 from T.mannum, 1 from T.capitis. 
T.verrucosum is isolated from 3 cases of $\mathrm{T}$. capitis. T.tonsurans from 2 cases of T.capitis. One isolate of Epidermophyton and one isolate of T.schoenlinii from T.capitis, one isolate of Microsporum gypseum from T.corporis.

\section{Discussion}

In our study more number of cases was seen in males when compared to females. Highest number of cases was seen in 1-10 years age group, followed by 21-30 years of age. Tinea corporis is the common clinical presentation of dermatophytosis in the present study which was in correlation with other studies from India by Peerapur et al, Singh et al, Parameswari. K et.al. ${ }^{6-8}$ Tinea capitis was common in children which were also observed in a study by Poluri et al. ${ }^{9}$ Trichophyton mentagrophytes was the most common dermatophyte followed by $\mathrm{T}$ rubrum to cause all clinical types of dermatophytoses. This was in correlation with study done by Sahai.s et al. ${ }^{5}$ Male predominance could be due to increased physical activities leading to excessive sweating, occupational exposure to infection and females attending less in number to clinic to seek treatment. Isolation rate is $32.6 \%$ which is low when compared to other studies. ${ }^{10-}$ 12 This could be due to non availability of fungal elements in the clinical material, factors involved in collection and inoculation of clinical material, culture conditions and severity of clinical manifestation. ${ }^{10}$

\section{Limitations}

Relatively smaller sample size and collection of single specimen from each patient are limitations of this study.

\section{Conclusion}

Dermatophytosis is a commonly seen superficial fungal infection in India because of hot and humid climate. In addition to early diagnosis and treatment, Health education regarding maintenance of personal hygiene, avoidance of tight clothing, various sources of transmission like exchange of clothes with infected individuals, use of hair brushes or combs of infected individuals, nail clippers, cotton caps and towels plays a major role in control of these infections. Dermatophyte species belonging to all 3 genera were isolated in the present study. Of the three genera of dermatophytes, Trichophyton species form the commonest etiological agent of dermatophytosis. In our study Tinea corporis was the common clinical type of all dermatophytosis followed by Tinea capitis. Trichophyton mentagrophytes is the frequently isolated species of dermatophyte followed by Trichophyton ruburm in this part of North Karnataka. Clinical diagnosis of these superficial skin infections are to be confirmed by direct microscopy and culture to differentiate them from other cases of dermatomycoses and to prevent recurrence of dermatophytosis by proper treatment.

\section{References}

1. Chander J. Textbook of Medical Mycology. 3rd ed. New Delhi: Mehta Publisher; 2009.

2. Kanwar AJ, Mamta, Chander J. Superficial fungal infections. In: Valia GR, editor. IADVL Text book and Atlas of Dermatology. 2nd ed. Mumbai: Bhalani Publishing House; 2001:215-58.

3. Tropical Dermatology, edited by Roberto Arenas and Roberto Estrada. (2001 Landes Bioscience.

4. Seebacher, C., Bouchara, J. P., \& Mignon, B. Updates on the epidemiology of dermatophyte infections. Mycopathologia, 2008;166(5-6):335-52.

5. Sahai Sanjeev, Mishra D. Change in spectrum of dermatophytes isolated from superficial mycoses cases: First report from central India. Ind J Dermatol Venereol Leprol 2011;77(3):335-6.

6. BV Peerapur, AC Inamdar, PV Pushpa, B Srikant. Ind J Med Microbiol 2004;22(4):273-74.

7. Singh S, Beena PM. Profile of dermatophyte infections in Baroda. Ind J Dermatol Venereol Leprol 2003;69:281-3.

8. Parameswari et al: Dermatophytosis in and around Kakinada. IJMDS 2015;4(2):828.

9. Poluri, L. V., Indugula, J. P., \& Kondapaneni, S.L. Clinicomycological study of dermatophytosis in South India. J Laboratory Physicians 2015;7(2):84.

10. Agarwal, U., Saran, J., \& Agarwal, P. Clinicomycological study of dermatophytes in a tertiary care centre in northwest India. Ind J Dermatol Venereol Leprol 2014;80(2):194.

11. Lakshmanan, A., Ganeshkumar, P., Mohan, S.R., Hemamalini, M., \& Madhavan, R. Epidemiological and clinical pattern of dermatomycoses in rural India. Ind $J$ Med Microbiol 2015;33(5):134.

12. Lone, R., Bashir, D., Ahmad, S., Syed, A., \& Khurshid, S. A study on clinicomycological profile, aetiological agents and diagnosis of onychomycosis at a government medical college hospital in Kashmir. J Clin Diagn Res: JCDR 2013;7(9):1983. 